\title{
Transient Global Cerebral Hypoperfusion as a Characteristic Cerebral Hemodynamic Pattern in the Acute Stage after Combined Revascularization Surgery for Pediatric Moyamoya Disease: N-Isopropyl-P-[ ${ }^{123}$ I] lodoamphetamine Single-Photon Emission Computed Tomography Study
}

\author{
Atsushi Kanoke ${ }^{a, b}$ Miki Fujimura ${ }^{a, c, d}$ Ryosuke Tashiro ${ }^{b}$ Dan Ozaki $^{b}$ \\ Teiji Tominagab \\ ${ }^{a}$ Department of Neurosurgery, Kohnan Hospital, Sendai, Japan; ${ }^{\mathrm{b}}$ Department of Neurosurgery, Tohoku University \\ Graduate School of Medicine, Sendai, Japan; 'Department of Neurosurgery, Hokkaido University Graduate School of \\ Medicine, Sapporo, Japan; 'Division of Advanced Cerebrovascular Surgery, Tohoku University Graduate School of \\ Medicine, Sendai, Japan
}

\section{Keywords}

Moyamoya disease $\cdot$ Pediatric patients - Revascularization surgery · 123I-IMP SPECT · Global hypoperfusion

\begin{abstract}
Introduction: Surgical revascularization prevents cerebral ischemic attack by improving cerebral blood flow (CBF) in both adult and pediatric patients with moyamoya disease (MMD). Uneven hemodynamic changes, including local cerebral hyperperfusion and remote ischemia, can cause delayed intracerebral hemorrhage and perioperative infarctions in adult MMD patients, but the characteristic hemodynamic pattern among pediatric MMD patients after revascularization surgery is poorly understood. Methods: This study included 16 consecutive pediatric MMD patients (age, 6-16 years; mean age, 11.3) undergoing superficial temporal artery-middle ce-
\end{abstract}

karger@karger.com www.karger.com/ced

Karger ${ }^{\prime \prime}$

BOPEN ACCESS
(C) 2021 The Author(s)

Published by S. Karger AG, Basel

This is an Open Access article licensed under the Creative Common Attribution-NonCommercial-4.0 International License (CC BY-NC) (http://www.karger.com/Services/OpenAccessLicense), applicable to the online version of the article only. Usage and distribution for commercial purposes requires written permission. rebral artery anastomosis combined with encephalo-duromyo-synangiosis on 21 affected hemispheres. Perioperative management was conducted by aspirin administration and strict blood pressure control $(110-130 \mathrm{~mm} \mathrm{Hg})$. We prospectively performed $\mathrm{N}$-isopropyl-p-[ $\left.{ }^{[23} \mathrm{l}\right]$ iodoamphetamine single-photon emission computed tomography on postoperative days (POD) 1 and 7 and analyzed the temporal changes in perioperative hemodynamics. Results: Four patients $(19.0 \%, 4 / 21)$ exhibited immediate CBF improvement from POD 1, which was classified as "immediate redistribution pattern." In contrast, $9(42.9 \%, 9 / 21)$ demonstrated transient hemispheric global hypoperfusion at POD 1 and subsequent CBF improvement at POD 7, which was defined as "transient hypoperfusion pattern." Although 8 patients, including 4 with "transient hypoperfusion pattern" $(44.4,4 / 9)$, developed mild transient neurological deterioration in the acute stage, it resolved in all 21 patients, and there were no permanent
Correspondence to:

Miki Fujimura, fujimur@med.hokudai.ac.jp 
neurological deficits. Discussion/Conclusions: This study revealed that the "transient hypoperfusion pattern" after revascularization surgery is relatively common among pediatric MMD patients, and its outcome is favorable under strict perioperative management.

(c) 2021 The Author(s)

Published by S. Karger AG, Basel

\section{Introduction}

Moyamoya disease (MMD) is a progressive steno-occlusive cerebrovascular disease at the terminal portion of the internal carotid artery and the development of moyamoya vessels $[1,2]$. It is an important cerebrovascular disease that can cause both ischemic and hemorrhagic stroke among young adults and children [1-3]. Surgical revascularization is effective to prevent the recurrence of ischemic attack and posterior hemorrhagic stroke in MMD [3]. Although several surgical managements, such as direct, indirect, and combined direct-indirect revascularization surgery, have been performed worldwide [46], we performed combined direct-indirect revascularization surgery for both adult and pediatric MMD patients and achieved good outcomes $[7,8]$.

The postoperative hemodynamics after combined direct-indirect bypass for pediatric MMD patients remain largely unknown. Among adult MMD patients, cerebral hyperperfusion (CHP) syndrome is one of the most deleterious complications after STA-MCA bypass surgery [9-16]. A hemodynamics study using $\mathrm{N}$-isopropyl-p$\left[{ }^{123} \mathrm{I}\right]$ iodoamphetamine single-photon emission computed tomography ( ${ }^{123}$ I-IMP SPECT) revealed that $56.9 \%$ of adult MMD patients have a characteristic hemodynamic pattern such as a transient increase in focal cerebral blood flow (CBF) on postoperative day (POD) 1 and subsequent distribution of $\mathrm{CBF}$ in the entire vascular territory on POD 7 [17]. CHP is known to result in transient neurological deficits, headache, seizure, and intracerebral hemorrhage in adult MMD patients [1016]. In addition, CHP can accompany the "watershed shift phenomenon," which is defined as paradoxical hypoperfusion in the adjacent cortex near the site of CHP $[18,19]$. To prevent the deleterious consequences of CHP, previous studies reported the effectiveness of strict blood pressure control and administration of minocycline hydrochloride $[20,21]$. However, the postoperative hemodynamics among pediatric MMD patients after combined direct-indirect bypass are poorly understood. In this study, we aimed to clarify the characteristic cerebral hemodynamic changes in the acute stage after combined direct-indirect revascularization surgery for pediatric MMD patients who were treated under a modern perioperative management protocol.

\section{Materials and Methods}

\section{Enrollment of Patients}

This case-control study was approved by the Ethics Committee of Kohnan hospital, Sendai, Japan, with the certification number of 2020-0520-02. Informed consent from all the patients was obtained. Sixteen consecutive pediatric patients with MMD (21 hemispheres; age, $6-16$ years; mean age, 11.3 ) surgically treated by a single neurosurgeon (M.F.) between March 2013 and May 2019 were enrolled in the current study. The diagnosis of MMD was determined based on magnetic resonance (MR) imaging and MR angiography according to the diagnostic criteria of the Research Committee on Spontaneous Occlusion of the Circle of Willis of the Ministry of Health, Labour, and Welfare, Japan [1, 2]. In some cases, the diagnosis was reinforced by catheter angiography. Surgical indications were considered if patients met all the following criteria: the presence of ischemic symptoms (minor completed stroke and/or TIAs), the presence of hemodynamic compromise on ${ }^{123}$ I-IMP-SPECT, independent activities of daily living (modified Rankin scale scores of $0-2$ ), and absence of major brain insults greater than the vascular territory of one major branch of the MCA.

\section{Surgical Procedures}

All patients underwent combined direct-indirect revascularization surgery by superficial temporal artery (STA)-middle cerebral artery (MCA) anastomosis with encephalo-duro-myo-synangiosis (EDMS), as described previously [7, 8, 10-12, 14-21]. After craniotomy around the Sylvian fissure, the stump of the STA was anastomosed to the cortical branch (M4 segment) of the MCA, followed by EDMS. To avoid compression of the brain surface by inserted temporal muscle, we routinely drilled out the inner layer of the bone flap and created a large bone window on the side of the EDMS flap. We confirmed the patency of STA-MCA bypass and no evidence of clot formation by intraoperative indocyanine green (ICG) video angiography and Doppler ultrasonography during all surgeries.

\section{Perioperative Radiological Evaluations}

Postoperative computed tomography (CT) was routinely performed immediately after the surgery and on POD 1 for all patients. MR imaging, including diffusion-weighted imaging, T2weighted imaging, and $\mathrm{T} 2 *$-weighted imaging, and MR angiography were performed on POD 2 or 3. The temporal profile of the postoperative cerebral hemodynamics was assessed by ${ }^{123} \mathrm{I}$-IMPSPECT. Quantitative analysis by the autoradiographic method was performed, if available. Postoperative temporal changes in $\mathrm{CBF}$ were classified into 3 patterns: first, the "transient hypoperfusion pattern" was defined as global hemispheric hypoperfusion on POD 1, which was considered somewhat paradoxical after STAMCA anastomosis, and subsequent improvement of CBF in the operated hemisphere on POD 7 (Fig. 1a). Decreased CBF values were confirmed by quantitative CBF measurement in some cases 
Fig. 1. Temporal changes in perioperative hemodynamics evaluated by ${ }^{123}$ I-IMPSPECT. a "Transient hypoperfusion pattern," represented by global hypoperfusion in the operated (right) side on POD 1 and improvement of CBF on POD 7. b "Immediate redistribution pattern," represented by improvement of CBF in the operated hemisphere (right) on POD 1 and recovery of CBF persisting until POD 7. ${ }^{123}$ I-IMPSPECT, N-isopropyl-p-[ $\left.{ }^{123} \mathrm{I}\right]$ iodoamphetamine single-photon emission computed tomography; POD, postoperative day; CBF, cerebral blood flow.

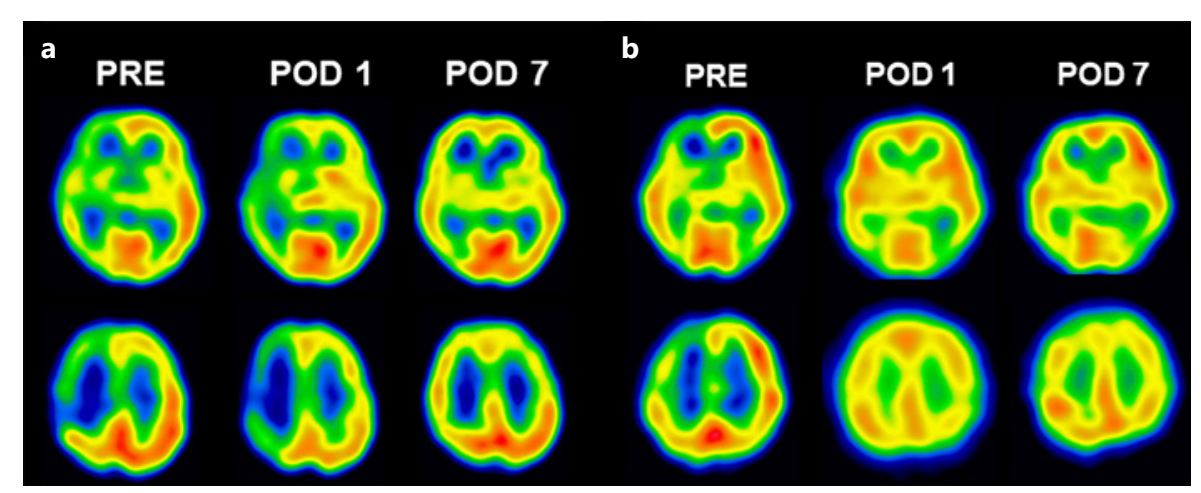

who can be subjected to quantitative SPECT studies by the autoradiographic method (online suppl. Fig. 1; for all online suppl. material, see www.karger.com/doi/10.1159/000520801). Second, the "immediate redistribution pattern" was defined as global but not focally excessive CBF improvement on POD 1 that persisted until POD 7 (Fig. 1b). Other patterns that met neither the "transient hypoperfusion pattern" nor the "immediate redistribution pattern" were defined as "others." The temporal postoperative changes in CBF were evaluated by 3 investigators independently (M.F., A.K., and R.T.).

\section{Perioperative Management Protocol}

All patients who underwent operations on 21 hemispheres were prospectively subjected to prophylactic intensive blood pressure reduction (systolic blood pressure of 100-130 mm Hg) according to a standardized postoperative management protocol to prevent CHP by continuous intravenous infusion of nicardipine hydrochloride, as reported previously $[9,10,14-18,20]$. In brief, all patients were subjected to intraoperative and postoperative administration of minocycline hydrochloride $(200 \mathrm{mg} /$ day $)$ until POD 5 to avoid the deleterious results of $\mathrm{CHP}$ and to reduce the potential risk of hemodynamic ischemia due to the watershed shift phenomenon. We routinely administered antiplatelet agents (100 mg aspirin/day) starting from POD 1 to all patients to avoid the unfavorable effects of intensive blood pressure reduction on the contralateral hemisphere and/or ipsilateral remote areas. Based on the temporal profile of ${ }^{123} \mathrm{I}$-IMP-SPECT, MR imaging, and MR angiography findings, we gradually allowed a return to normotensive blood pressure between POD 3 and 7.

\section{Results}

Temporal changes in perioperative hemodynamics were evaluated using ${ }^{123}$ I-IMP-SPECT. The postoperative temporal changes on ${ }^{123}$ I-IMP-SPECT were classified into 3 patterns: "transient hypoperfusion pattern," "immediate redistribution pattern," and "others," as shown in Figure 1. The temporal changes in postoperative hemodynamics in each patient are summarized in Table 1.
There were no cases who required sedation during SPECT studies. The "immediate redistribution pattern" was noted in 4 hemispheres $(19.0 \%, 4 / 21)$. The "transient hypoperfusion pattern" was evident in 9 hemispheres $(42.9 \%$, 9/21). The "transient hypoperfusion pattern" was evident in their 1st operation in 7 hemispheres $(77.8 \%, 7 / 9)$ and 2nd operation (surgery for contralateral hemisphere of 1 st operation) in 2 hemispheres $(22.2 \%, 2 / 9)$. The temporal postoperative changes in 8 hemispheres were classified into "others" $(38.1 \%, 8 / 21)$. Among patients whose SPECT findings were classified as "others," 2 patients demonstrated global hypoperfusion in operated hemisphere at POD 1 and 7 but CBF improvement at chronic stages (cases 14 and 15), 2 patients showed similar CBF levels as preoperative values at POD 1 and 7 (cases 16 and 21), 1 patient showed slight CBF improvement at POD 1 (case 17), 2 patients showed CBF improvement at POD 7 (cases 18 and 20), and 1 patient showed similar CBF values at POD 1 and 7 but demonstrated CBF improvement at chronic stage (case 19). No patients developed focal CHP. Although 8, including 4 with the "transient hypoperfusion pattern" $(44.4 \%, 4 / 9), 1$ with the "immediate redistribution pattern" $(25.0 \%, 1 / 4)$, and 3 with "gradual redistribution pattern" $(37.5 \%, 3 / 8)$, developed mild transient neurological deterioration in the acute stage, it resolved in all 8 hemispheres, and there were no neurological deficits.

\section{Representative Case (Case 3)}

An 8-year-old girl was referred to our institution with a history of transient paralysis of the left extremities while crying. One of her grandparents had a history of MMD. MR angiography demonstrated markedly stenotic lesions at the terminal portion of the bilateral internal carotid 
Table 1. The list of patients included in this study

\begin{tabular}{|c|c|c|c|c|c|c|c|c|c|}
\hline Surgery & Age & Sex & Onset & $\begin{array}{l}\text { Op } \\
\text { side }\end{array}$ & $\begin{array}{l}1 \mathrm{st} / \\
2 \mathrm{nd} \text { op }\end{array}$ & $\begin{array}{l}\text { STA } \\
\text { diameter, } \\
\mathrm{mm}\end{array}$ & $\begin{array}{l}\text { Occlusion } \\
\text { time }\end{array}$ & ${ }^{123}$ I-IMP SPECT findings & Postoperative neurological deficits \\
\hline 1 & 16 & $\mathrm{~F}$ & 1 & $\mathrm{~L}$ & 2nd & 1 & 28 & Transient hypoperfusion & - \\
\hline 2 & 12 & $\mathrm{~F}$ & I & $\mathrm{R}$ & $1 \mathrm{st}$ & 1.2 & 25 & Transient hypoperfusion & - \\
\hline 3 & 8 & $\mathrm{~F}$ & 1 & $\mathrm{R}$ & $1 \mathrm{st}$ & 1 & 19 & Transient hypoperfusion & $\begin{array}{l}\text { Transient paralysis of right } \\
\text { extremities after crying at POD } 1\end{array}$ \\
\hline 4 & 9 & $\mathrm{~F}$ & 1 & $\mathrm{R}$ & $1 \mathrm{st}$ & 1 & 17 & Transient hypoperfusion & $\begin{array}{l}\text { Transient paralysis of right and left } \\
\text { extremities after crying at POD } 7\end{array}$ \\
\hline 5 & 9 & $\mathrm{~F}$ & 1 & $\mathrm{R}$ & $1 \mathrm{st}$ & 0.6 & 19 & Transient hypoperfusion & - \\
\hline 6 & 9 & $\mathrm{~F}$ & 1 & $\mathrm{~L}$ & 2nd & 0.8 & 20 & Transient hypoperfusion & $\begin{array}{l}\text { Transient paralysis of right } \\
\text { extremities after crying at POD } 12\end{array}$ \\
\hline 7 & 12 & $\mathrm{~F}$ & 1 & $\mathrm{~L}$ & $1 \mathrm{st}$ & 1 & 20 & Transient hypoperfusion & $\begin{array}{l}\text { Transient paralysis of right } \\
\text { extremities at POD } 1 \text { and } 2\end{array}$ \\
\hline 8 & 9 & M & 1 & $\mathrm{R}$ & $1 \mathrm{st}$ & 0.8 & 28 & Transient hypoperfusion & - \\
\hline 9 & 7 & $\mathrm{~F}$ & 1 & $\mathrm{R}$ & $1 \mathrm{st}$ & 0.7 & 27 & Transient hypoperfusion & - \\
\hline 10 & 14 & $\mathrm{~F}$ & 1 & $\mathrm{~L}$ & $1 \mathrm{st}$ & 1 & 26 & Immediate redistribution & - \\
\hline 11 & 13 & $\mathrm{~F}$ & 1 & $\mathrm{~L}$ & $1 \mathrm{st}$ & 1.2 & 27 & Immediate redistribution & $\begin{array}{l}\text { Transient aphasia and paralysis of } \\
\text { right extremities at POD } 3 \text { and } 4\end{array}$ \\
\hline 12 & 14 & $\mathrm{~F}$ & 1 & $\mathrm{R}$ & 2nd & 1 & 25 & Immediate redistribution & - \\
\hline 13 & 16 & $\mathrm{~F}$ & 1 & $\mathrm{R}$ & 2nd & 1 & 24 & Immediate redistribution & - \\
\hline 14 & 12 & $\mathrm{~F}$ & 1 & $\mathrm{~L}$ & 2nd & 0.9 & 21 & $\begin{array}{l}\text { Others (global hypoperfusion at acute stage } \\
\text { and CBF improvement at chronic stage) }\end{array}$ & - \\
\hline 15 & 12 & $\mathrm{~F}$ & I & $\mathrm{R}$ & $1 \mathrm{st}$ & 1 & 20 & $\begin{array}{l}\text { Others (global hypoperfusion at acute stage } \\
\text { and CBF improvement at chronic stage) }\end{array}$ & $\begin{array}{l}\text { Transient paralysis of left } \\
\text { extremities after crying at POD } 3\end{array}$ \\
\hline 16 & 8 & $\mathrm{~F}$ & I & $\mathrm{L}$ & 2nd & 1 & 20 & Others (same as preop) & \\
\hline 17 & 13 & $\mathrm{~F}$ & I & $\mathrm{L}$ & 2nd & 0.8 & 21 & Others (slight CBF improvement at POD 1) & \\
\hline 18 & 14 & $\mathrm{~F}$ & I & $\mathrm{R}$ & $1 \mathrm{st}$ & 1 & 22 & Others (slight CBF improvement at POD 7) & $\begin{array}{l}\text { Transient paralysis of right } \\
\text { extremities at POD } 2 \text { and left } \\
\text { extremities at POD } 3\end{array}$ \\
\hline 19 & 16 & $\mathrm{~F}$ & I & $\mathrm{L}$ & $1 \mathrm{st}$ & 0.8 & 23 & Others (CBF improvement at chronic stage) & $\begin{array}{l}\text { Transient paralysis of right } \\
\text { extremities at POD } 11\end{array}$ \\
\hline 20 & 9 & $\mathrm{~F}$ & I & $\mathrm{L}$ & $1 \mathrm{st}$ & 0.8 & 26 & Others (CBF improvement at POD 7) & - \\
\hline 21 & 6 & $\mathrm{~F}$ & I & $\mathrm{R}$ & $1 \mathrm{st}$ & 1.2 & 18 & Others (same as preop) & - \\
\hline
\end{tabular}

The 2nd operation (2nd op) indicates the bypass surgery for contralateral hemisphere of the 1st operation. M, male; F, female; Op, operation; I, ischemia; $\mathrm{R}$, right; $\mathrm{L}$, left; STA, superficial temporal artery; POD, postoperative day; ${ }^{123}$-IMP SPECT, N-isopropyl-p- $\left[{ }^{123}\right]$ ] iodoamphetamine single-photon emission computed tomography; $\mathrm{CBF}$, cerebral blood flow.

arteries, leading to the definite diagnosis of MMD (Fig. 2a-b). She had no neurological deficits or medical history. ${ }^{123}$ I-IMP SPECT demonstrated decreased CBF in her right hemisphere. She underwent right combined direct-indirect revascularization surgery by STA-MCA anastomosis and EDMS. Intraoperative ICG video angiography and Doppler ultrasonography confirmed patency of the right STA-MCA bypass, and the bypass flow was well distributed in the entire operated hemisphere
(Fig. 2c-d). There was no evidence of clot formation in donor or recipient arteries on intraoperative ICG video angiography (Fig. 2c). Although patency of the STAMCA bypass was confirmed, ${ }^{123}$ I-IMP-SPECT 1 day after surgery revealed global hypoperfusion in the entire right hemisphere (Fig. 2e). Postoperative MR angiography 2 days after surgery further confirmed patency of the right STA-MCA bypass (Fig. 2f). Postoperative MR imaging 2 days after surgery also demonstrated no evidence of cere- 


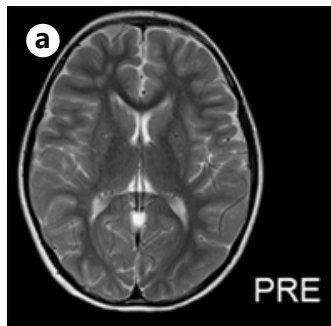

b PRE

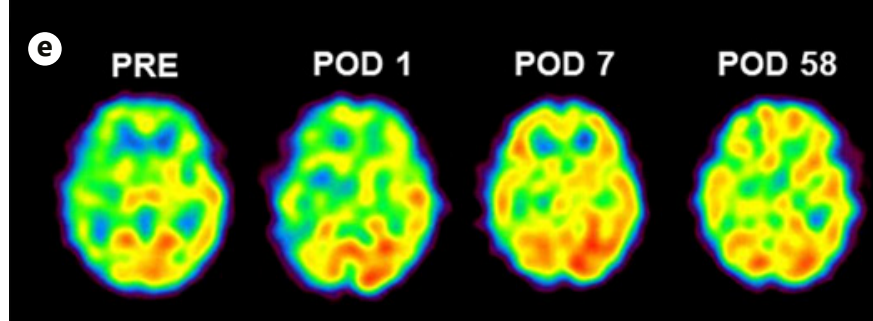

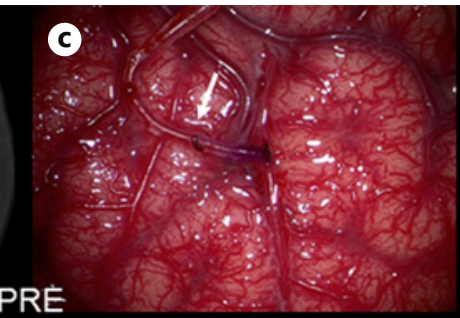

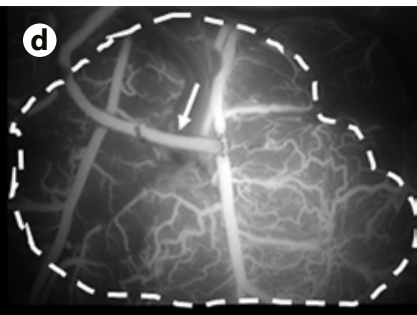

(9)

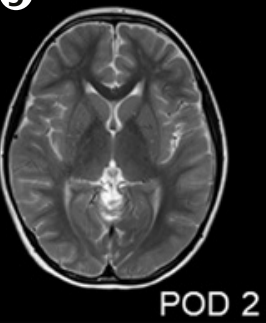

(h

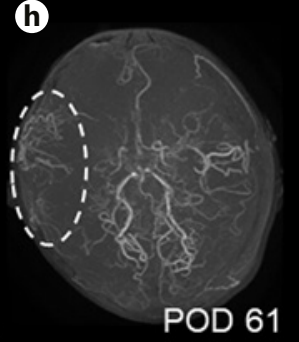

(i)

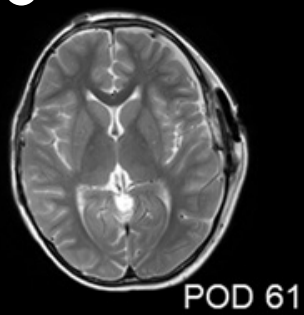

Fig. 2. Representative case (case 3 ) demonstrating the "transient hypoperfusion pattern." An 8-year-old patient with ischemic symptoms underwent right STA-MCA anastomosis with indirect vascularization surgery. a Preoperative T2-weighted imaging showed no evidence of infarction. b Preoperative MR angiography confirmed the definite diagnosis of MMD. c Intraoperative view of the right STA-MCA bypass (arrow). There was no evidence of intravascular clots in donor or recipient arteries. Arrow indicate the STA. d Intraoperative ICG video angiography confirmed the patency of the STA-MCA bypass and no evidence of clot formation in the donor and recipient arteries. The arrow and dotted line indicate the STA and distribution of bypass flow, respectively. e Temporal changes in perioperative cerebral blood flow evaluated

bral infarctions or compression of the brain surface (Fig. $2 \mathrm{~g}$ ). As the patient had no signs of ischemic symptoms, we continued standardized perioperative management, including strict blood pressure control and administration of minocycline hydrochloride. ${ }^{123} \mathrm{I}$-IMP-SPECT on POD 7 revealed significant improvement of $\mathrm{CBF}$ in her entire right hemisphere (Fig. 2e). Although the patient experienced transient paralysis of the right extremities after crying aloud on POD 1, she was free from permanent neurological deficits. Then, 2 months later, the patient underwent left STA-MCA anastomosis with EDMS. Postoperative MR angiography 2 days after left revascularization surgery confirmed the patency of the by ${ }^{123}$ I-IMP-SPECT, showing global hypoperfusion on POD 1 and gradual improvement of CBF on POD 7 and 61. f Postoperative MR angiography confirming the patency of the right STA-MCA bypass (arrow) on POD 2. g Postoperative T2-weighted imaging on POD 2 confirming no ischemic lesions in the right hemisphere. h Postoperative MR angiography on POD 61 confirming the patency of the right STA-MCA bypass and the development of pial synangiosis (dotted line). i Preoperative T2-weighted imaging on POD 61 showed no evidence of ischemic lesions. STA-MCA, superficial temporal artery-middle cerebral artery; $\mathrm{MR}$, magnetic resonance; MMD, moyamoya disease; ICG, indocyanine green; $\mathrm{POD}$, postoperative day; $\mathrm{CBF}$, cerebral blood flow.

STA-MCA bypass and the development of pial synangiosis (Fig. 2h). Postoperative MR imaging also confirmed no ischemic lesions in either hemisphere (Fig. 2i). The postoperative course after left revascularization surgery was uneventful, and the patient is doing well without recurrent ischemic attack.

\section{Discussion}

The present study revealed that the unique "transient hypoperfusion pattern" is relatively common in pediatric patients with MMD after combined direct-indirect revas- 
cularization surgery. Our study also demonstrated that the outcome of this unique hemodynamic pattern is generally favorable.

Postoperative global hypoperfusion may present as unique hemodynamic changes among pediatric MMD patients. Among adult patients, CHP is one of the major hemodynamic issues that can result in not only transient neurological deficits, severe headaches, and seizures but also delayed intracerebral hemorrhage [9-16]. Based on previous studies, the incidence of radiological and symptomatic CHP among adult MMD patients is as high as $27.8-56.9 \%$ and $0-38.2 \%$, respectively $[10-17,19]$. In contrast, none of the pediatric patients had CHP, either radiological or symptomatic, in the current study. This is consistent with previous reports stating that the incidence of CHP is lower in pediatric patients than in adults $[7,8,22]$. Our study further revealed that the "transient hypoperfusion pattern" is the most common hemodynamic pattern, which was observed in $42.9 \%$ of pediatric MMD patients after combined direct-indirect revascularization surgeries. Moreover, the "transient hypoperfusion pattern" might be more common in 1st operation compared with 2 nd operation for contralateral side of $1 \mathrm{st}$ operation (Table 1). On the other hand, similar postoperative hemodynamic changes are rarely observed in adult MMD patients $[6,13,17]$. These observations support the notion that postoperative hemodynamics can differ among pediatric and adult MMD patients.

The exact mechanism underlying global hypoperfusion among pediatric MMD patients remains unknown. Ischemic complications after STA-MCA bypass may be caused by at least 3 distinct mechanisms: the "watershed shift phenomenon" $[18,19]$, thromboembolism at the site of the anastomosis [12], and mechanical compression of the brain surface by swollen temporal muscle used for the indirect bypass procedure [23]. In the current study, we confirmed the patency of the STA-MCA bypass in all patients by intraoperative video ICG angiography (Fig. 2d) and Doppler sonography and postoperative MR angiography (Fig. 2f, h). Postoperative MR imaging confirmed that there was no mechanical compression of the brain surface by the inserted temporal muscle. In addition, no patient developed focal CHP, which may accompany hemodynamic ischemia due to the watershed shift phenomenon. Therefore, postoperative global hypoperfusion may be due to a specific pathophysiology in pediatric MMD patients.

Intrinsic vascular fragility and potential inflammationprone environment in pediatric MMD cases might contribute to this unique hemodynamics in pediatric MMD patients. Takagi et al. [24] investigated the difference in histopathological findings of cerebral arteries among adult and pediatric MMD patients. They found the intima of MCA was thicker in adult MMD patients compared to pediatric MMD patients [24]. This histopathological difference in cerebral arteries might be responsible for the vulnerability to mechanical stimuli, including surgical manipulation, in pediatric MMD patients. In addition, the inserted temporal muscle flap could induce vasospasm at the acute stage of combined direct-indirect bypass surgery. Given that neovascularization after indirect bypass surgery is more common in pediatric MMD cases compared with adult MMD cases [25-27], the temporal muscle flap in pediatric cases might produce more angiogenic and trophic factors and induce stronger inflammation compared to adult cases. Even though we confirmed the apparent patency of the STA-MCA bypass by intraoperative ICG video angiography and Doppler ultrasonography and postoperative MR angiography, the intrinsic vascular vulnerability and potential inflammation-prone state may easily induce vasospasm of donor and/or recipient arteries after combined direct-indirect revascularization surgery for pediatric cases.

The outcome of global hypoperfusion among pediatric patients is generally favorable. Under strict perioperative management by routine assessment of perioperative hemodynamics using ${ }^{123}$ I-IMP SPECT, blood pressure control (systolic blood pressure of 100-130 mm Hg), and administration of neuroprotective/anti-inflammatory agents (minocycline hydrochloride) and aspirin [20,21], none of the pediatric patients with the "transient hypoperfusion pattern" developed permanent ischemic complications, although several patients exhibited transient ischemic symptoms after crying (Table 1). However, in a previous study that investigated the postoperative cerebral hemodynamics using flow probes, local hypoperfusion was correlated with transient neurological deficits [28]. In addition, local hypoperfusion may be associated with vasogenic edema, which may result from intrinsic vascular vulnerability in MMD patients [29]. As such, although close monitoring is necessary, the outcome of transient global hypoperfusion after combined direct-indirect revascularization surgery for pediatric MMD patients may be favorable under strict management. Alternatively, excessive blood pressure reduction should be avoided in pediatric MMD patients to prevent ischemic complications during the state of global cerebral hypoperfusion. Given these observations, we may allow blood pressure increase for the pediatric cases who demonstrated the "transient hypoperfusion pattern." Despite the need for further investigations, perioperative management protocols for pe- 
diatric MMD cases should be optimized based on the unique hemodynamics among pediatric MMD patients.

There are several limitations of this retrospective study at a single institution. First, qualitative assessment of temporal hemodynamic changes on ${ }^{123}$ I-IMP-SPECT may yield an evaluation bias even though the data were evaluated by 3 independent investigators who confirmed the concordance of data interpretations. Despite the necessity of arterial blood sampling, serial quantitative SPECT studies are needed to evaluate the accurate temporal changes of CBF. Second, the incidence of the "transient hypoperfusion pattern" might be overestimated, since all the patients were managed strict blood pressure control under $130 \mathrm{~mm} \mathrm{Hg}$ until SPECT study at POD 1. Third, the number of patients included in the current study was relatively small. Fourth, long-term outcomes due to global hypoperfusion were not evaluated in the current study. Further studies including larger cohorts from multiple institutions that investigate long-term outcomes are warranted to validate the findings of the present study.

\section{Conclusions}

This study revealed unique hemodynamic changes after STA-MCA bypass in pediatric MMD patients. Approximately $40 \%$ of pediatric MMD patients demonstrated transient hemispheric global hypoperfusion on POD 1 , followed by the gradual improvement of CBF in both hemispheres. The outcome of the "transient hypoperfusion pattern" may be generally favorable under modern perioperative management.

\section{Statement of Ethics}

All procedures performed in this study were in accordance with the ethical standards of the institution and with the 1964 Helsinki Declaration and its later amendments or comparable ethical standards. The Ethics Committee of Kohnan Hospital approved this study (Approval No. 2020-0520-02). Informed consent was received from the guardians of individual participants included in the study.

\section{Conflict of Interest Statement}

All authors have no conflicts of interest to declare.

\section{Funding Sources}

This study was supported by JSPS KAKENHI Grant 20K09362.

\section{Author Contributions}

M.F. contributed to conception and design and acquisition of data. M.D., A.K., R.T., and D.O. contributed to analysis and interpretation. A.K. contributed to drafting. M.F., R.T., D.O., and T.T. contributed to critical revision of the article. T.T. contributed to study supervision.

\section{Data Availability Statement}

All data generated or analyzed during this study are included in this article. Further enquiries can be directed to the corresponding author.

\section{References}

1 Research Committee on the Pathology and Treatment of Spontaneous Occlusion of the Circle of Willis, Health Labour Sciences Research Grant for Research on Measures for Intractable Diseases. Guidelines for diagnosis and treatment of moyamoya disease (spontaneous occlusion of the circle of Willis). Neurol Med Chir. 2012;52(5):245-66.

2 Tominaga T, Suzuki N, Miyamoto S, Koizumi A, Kuroda S, Takahashi JC, et al. Recommendations for the management of Moyamoya disease: a statement from Research Committee on Spontaneous Occlusion of the Circle of Willis (Moyamoya Disease) [2nd Edition]. Surg Cereb Stroke. 2018;46(1):1-24.

3 Miyamoto S, Yoshimoto T, Hashimoto N, Okada Y, Tsuji I, Tominaga T, et al. Effects of extracranial-intracranial bypass for patients with hemorrhagic moyamoya disease: results of the Japan adult Moyamoya trial. Stroke. 2014;45(5):1415-21.

4 Abla AA, Gandhoke G, Clark JC, Oppenlander ME, Valet GJ, Zabramski JM, et al. Surgical outcomes for moyamoya angiopathy at barrow neurological institute with comparison of adult indirect encephaloduroarteriosynangiosis bypass, adult direct superficial temporal artery-to-middle cerebral artery bypass, and pediatric bypass: 154 revascularization surgeries in 140 affected hemispheres. Neurosurgery. 2013;73:430-9.

$5 \mathrm{Ha}$ EJ, Kim KH, Wang KC, Phi JH, Lee JY, Choi JW, et al. Long-term outcomes of indirect bypass for 629 children with Moyamoya disease: longitudinal and cross-sectional analysis. Stroke. 2019 Nov;50(11):3177-83.
6 Zheng J, Yu LB, Dai KF, Zhang Y, Wang R, Zhang D. Clinical features, surgical treatment, and long-term outcome of a multicenter cohort of pediatric Moyamoya. Front Neurol. 2019;10:14.

7 Fujimura M, Kaneta T, Tominaga T. Efficacy of superficial temporal artery-middle cerebral artery anastomosis with routine postoperative cerebral blood flow measurement during the acute stage in childhood moyamoya disease. Childs Nerv Syst. 2008;24(7):827-32.

8 Rashad S, Fujimura M, Noisome K, Endo H, Taming T. Long-term follow-up of pediatric moyamoya disease treated by combined direct-indirect revascularization surgery: single institute experience with surgical and postoperative management. Neurosurgeon Rev. 2016;39(4):615-523. 
9 Kim JE, Oh CW, Kwon OK, Park SQ, Kim SE, Kim YK. Transient hyperperfusion after superficial temporal artery/middle cerebral artery bypass surgery as a possible cause of postoperative transient neurological deterioration. Cerebrovasc Dis. 2008;25(6):580-6.

10 Fujimura M, Mugikura S, Kaneta T, Shimizu $\mathrm{H}$, Tominaga $\mathrm{T}$. Incidence and risk factors for symptomatic cerebral hyperperfusion after superficial temporal artery-middle cerebral artery anastomosis in patients with moyamoya disease. Surg Neurol. 2009;71:442-7.

11 Fujimura M, Shimizu H, Mugikura S, Tominaga $T$. Delayed intracerebral hemorrhage after superficial temporal artery-middle cerebral artery anastomosis in a patient with moyamoya disease: possible involvement of cerebral hyperperfusion and increased vascular permeability. Surg Neurol. 2009;71(2): 223-7; discussion 227.

12 Fujimura M, Shimizu H, Inoue T, Mugikura S, Saito A, Tominaga T. Significance of focal cerebral hyperperfusion as a cause of transient neurologic deterioration after extracranial-ntracranial bypass for moyamoya disease: comparative study with non-moyamoya patients using N-isopropyl-p-[(123)I]iodoamphetamine single-photon emission computed tomography. Neurosurgery. 2011; 68(4):957-64; discussion 964-5.

13 Uchino H, Kuroda S, Hirata K, Shiga T, Houkin K, Tamaki N. Predictors and clinical features of postoperative hyperperfusion after surgical revascularization for moyamoya disease: a serial photon emission CT/positron tomography study. Stroke. 2012;43(10): 2610-6.

14 Kameyama M, Fujimura M, Tashiro R, Sato K, Endo H, Niizuma K, et al. Significance of quantitative cerebral blood flow measurement in the acute stage after revascularization surgery for moyamoya disease: implication for the pathological threshold of local cerebral hyperperfusion. Cerebrovsc Dis. 2019;48(36):217-25.

15 Nishizawa T, Fujimura M, Katsuki M, Mugikura S, Tashiro R, Sato K, et al. Prediction of cerebral hyperperfusion after superficial temporal artery-middle cerebral artery anastomosis by three-dimensional-time-of-flight magenetic resonance angiography in adult patients with Moyamoya disease. Cerebrovasc Dis. 2020;49(4):396-403.

16 Tashiro R, Fujimura M, Kastuki M, Nishizawa T, Tomata Y, Niizuma K, et al. Prolonged/ delayed cerebral hyperperfusion in adult patients with moyamoya disease with RNF213 gene polymorphism c.14576G $>\mathrm{A}$ (rs112735431) after superficial temporal artery-middle cerebral artery anastomosis. J Neurosurg. 2020 Oct 23:1-8. [online ahead of print].

17 Fujimura M, Tominaga T. Characteristic patterns of the cerebral hemodynamic changes in the acute stage after combined revascularization surgery for adult moyamoya disease: $\mathrm{N}$ isopropyl-p-[123I]iodoamphetamine singlephoton emission computed tomography study. Acta Neurochir Suppl. 2021;132:57-61.

18 Hayashi T, Shirane R, Fujimura M, Tominaga T. Postoperative neurological deterioration in pediatric moyamoya disease: watershed shift and hyperperfusion. J Neurosurg Pediatr. 2010 Jul 6;6(1):73-81.

19 Tashiro R, Fujimura M, Kameyama M, Mugikura S, Endo H, Takeuchi Y, et al. Incidence and risk factors of the watershed shift phenomenon after superficial temporal arterymiddle cerebral artery anastomosis for adult moyamoya disease. Cerebrovasc Dis. 2019; 47(3-4):178-87.

20 Fujimura $\mathrm{M}$, Inoue $\mathrm{T}$, Shimizu $\mathrm{H}$, Saito A, Mugikura S, Tominaga T. Efficacy of prophylactic blood pressure lowering according to a standardized postoperative management protocol to prevent symptomatic cerebral hyperperfusion after direct revascularization surgery for moyamoya disease. Cerebrovasc Dis. 2012;33(5):436-45.

21 Fujimura M, Niizuma K, Inoue T, Sato K, Endo $\mathrm{H}$, Shimizu H, et al. Minocycline prevents focal neurological deterioration due to cerebral hyperperfusion after extracranial-intracranial bypass for moyamoya disease. Neu- rosurgery. 2014 Feb;74(2):163-70; discussion 170

22 Kazumata K, Ito M, Tokairin K, Ito Y, Houkin $\mathrm{K}$. The frequency of postoperative stroke in moyamoya disease following combined revascularization: a single-university series and systemic review. J Neurosurg. 2014 Aug; 121(2):432-40.

23 Fujimura M, Kaneta T, Shimizu H, Tominaga T. Cerebral ischemia owing to compression of the brain by swollen temporal muscle used for encephalo-myo-synangiosis in moyamoya disease. Neurosurg Rev. 2009 Apr;32(2):2459; discussion 249.

24 Takagi Y, Hermanto Y, Takahashi JC, Funaki T, Kikuchi T, Mineharu Y, et al. Histopathological characteristics of distal middlecrerbral artery in adult and pediatric patients with moyamoya disease. Neurol Med Chir. 2016; 56:345-9.

25 Mizoi K, Kayama T, Yoshimoto T, Nagamine Y. Indirect revascularization for moyamoya disease: is there a beneficial effect for adult patients? Surg Neurol. 1996;45:541-9.

26 Houkin K, Kuroda S, Ishikawa T, Abe H. Neovascularization (angiogenesis) after revascularization in Moyamoya disease. Which technique is most useful for Moyamoya disease? Acta Neurochir. 2000;142:269-76.

27 Uchino H, Kim JH, Fujima N, Kazumata K, Ito $\mathrm{M}$, Nakayama N, et al. Synergistic interactions between direct and indirect bypasses in combined procedures: the significance of indirect bypasses in moyamoya disease. Neurosurgery. 2017;80:201-9.

28 Mukerji N, Cook DJ, Steinberg GK. Is local hypoperfusion the reason for transient neurological deficits after STA-MCA bypass for moyamoya disease? J Neurosurg. 2015 Jan; 122(1):90-4.

29 Tashiro R, Fujimura M, Mugikura S, Niizuma $\mathrm{K}$, Endo H, Endo T, et al. Paradoxical association of symptomatic local vasogenic edema with global cerebral hypoperfusion after direct revascularization surgery for adult Moyamoya disease. J Stroke Cerebrovasc Dis. 2018 Aug;27(8):e172-e176. 